\title{
Study of the stability in holographic reflection gratings recorded in PVA/AA based photopolymer
}

\author{
Elena Fernández $z^{1,3^{*}}$, Rosa Fuentes ${ }^{1,3}$, Manuel Ortuño ${ }^{2,3}$, Andres Marquez ${ }^{2,3}$, Augusto Beléndez ${ }^{2,3}$ \\ and Inmaculada Pascual ${ }^{2,3}$ \\ ${ }^{1}$ Departamento de Óptica, Farmacología y Anatomía, Universidad de Alicante, Apartado 99, E- \\ 03080 Alicante, Spain \\ ${ }^{2}$ Departamento de Física, Ingeniería de Sistemas y Teoría de la Señal, Universidad de Alicante, \\ Apartado 99, E-03080 Alicante, Spain \\ ${ }^{3}$ Instituto Universitario de Física Aplicada a las Ciencias y las Tecnologías, Universidad de \\ Alicante, Ap.Correos 99, E-03080 Alicante \\ *E-mail: elena.fernandez@ua.es
}

\begin{abstract}
Holographic reflection gratings were stored in a PVA/AA based photopolymer material using symmetrical geometry. Diffraction efficiency of the gratings was measured and a curing process was applied in the gratings to fix them. The aim of this paper is to analyze the stability of stored gratings with over time, after to apply the curing process, comparing the results obtained with and without curing.
\end{abstract}

Keywords: Photopolymers, reflection holograms, stability of the gratings.

\section{INTRODUCTION}

In the last decade holography has acquired great importance since devices can store the information in the entire volume of the material ${ }^{1-4}$, thereby increasing the storage capacity in comparison with two-dimensional devices that only store the information on the surface. Pioneering companies in this field, such as Bayer MaterialScience and InPhase ${ }^{3,4}$ even came together to create the TapestryTM, the first prototype of holographic optical storage system that is being used by leading companies and is capable of storing from 200 Gbytes to 1.6 Tbytes in a disk of $130 \mathrm{~mm}$ in diameter.

The most widely studied branch of holography, which has given rise to a large number of papers, is transmission holography ${ }^{5}$. However, leading companies such as Bayer are beginning to conduct studies aimed at using reflection holography ${ }^{6}$. In order to obtain more compact systems it would be interesting to design reflection holographic memories to which the current technology for reading CD's and DVD's designed for reflection holograms may be applied.

In this study reflection holographic gratings are recorded using a symmetric geometry in a PVA/AA photopolymer ${ }^{7,8}$. The photopolymer is composed of acrylamide (AA) as the polymerizable monomer, triethanolamine (TEA) as radical generator, N,N'-methylene-bis-acrylamide (BMA) as cross-linker, yellowish eosin (YE) as sensitizer and a binder of polyvinyl alcohol (PVA). The resulting layers are about $70 \mu \mathrm{m}$ thick.

Optical Modelling and Design II, edited by Frank Wyrowski, John T. Sheridan, Jani Tervo, Youri Meuret, Proc. of SPIE Vol. 8429, 84291Z - (c) 2012 SPIE · CCC code: 0277-786X/12/\$18 · doi: 10.1117/12.921636 
Once the holographic reflection gratings are stored, the transmittance of the grating will be measured versus the readout wavelength using a double-beam spectrophotometer at normal incidence in order to obtain their diffraction efficiency.

In this work the changes in the diffraction efficiency as a function of time elapsed since the recording until the moment of analysis of the grating are studied, and some methods of fixing to reduce the changes of this parameter with time will be applied.

\section{EXPERIMENTAL}

\subsection{Preparation of the material}

The holograms are recorded in a photopolymer composed of acrylamide (AA) as the polymerizable monomer, triethanolamine (TEA) as radical generator, N,N'-methylene-bis-acrylamide (BMA) as cross-linker, yellowish eosin (YE) as sensitizer and a binder of polyvinyl alcohol (PVA).

Table 1 shows the component concentrations of the photopolymer composition used to obtain layers about $70 \mu \mathrm{m}$ thick.

Table 1. Concentrations of the Photopolymer composition

\begin{tabular}{cc}
\hline & Composition \\
\hline Polyvinylalcohol (PVA) & $8.3 \% \mathrm{~m} / \mathrm{v}$ \\
Acrylamide (AA) & $0.33 \mathrm{M}$ \\
Triethanolamine (TEA) & $0.19 \mathrm{M}$ \\
Yellowish eosin (EA) & $2.45 \cdot 10^{-4} \mathrm{M}$ \\
N,N'methylene-bis-acrylamide \\
(BMA) \\
0
\end{tabular}

A solution of PVA in water forms the matrix and this is used to prepare the mixture of AA, BMA, and photopolymerization initiator system composed of TEA and YE. The mixture is made under red light, deposited by gravity on a $22 \mathrm{~cm} \times 40 \mathrm{~cm}$ glass plate and left in the dark for one day approximately to allow the water to evaporate in conditions of temperature, $\mathrm{T}$, between $18{ }^{\circ} \mathrm{C}$ and $20^{\circ} \mathrm{C}$, and relative humidity, $\mathrm{RH}, 30 \%-40 \%$. Once dry, the glass is cut into squares of $6 \times 6 \mathrm{~cm}^{2}$.

The refractive index of the material is calculated using an Abbe's refractometer and a refractive index of $\mathrm{n}=1.519$ was obtained for a wavelength of $532 \mathrm{~nm}$ and $\mathrm{n}=1.509$ for a wavelength of $653 \mathrm{~nm}$. These two refractive indexes will be used to calculate the spatial period of the recorded gratings and the Bragg wavelength in section 2.2.

Figure 1 shows the transmittance of the material versus the wavelength. As it can be observed, the plot has a minimum transmittance (maximum absorption) at the recording wavelength $\lambda=532 \mathrm{~nm}$. Consequently, the material composition is optimized so that the material is as sensitive as possible at the recording wavelength.

In the range between $\lambda=570 \mathrm{~nm}$ and $\lambda=590 \mathrm{~nm}$, the transmittance is maximum and thus the material does not absorb or absorbs very little. The geometries for the grating recording and reconstruction were chosen so that the Bragg wavelength would appear in such non absorbing spectral range (see section 2.2) in order to avoid overlapping of the Bragg and absorption peaks (such overlapping would probably mask the results). 


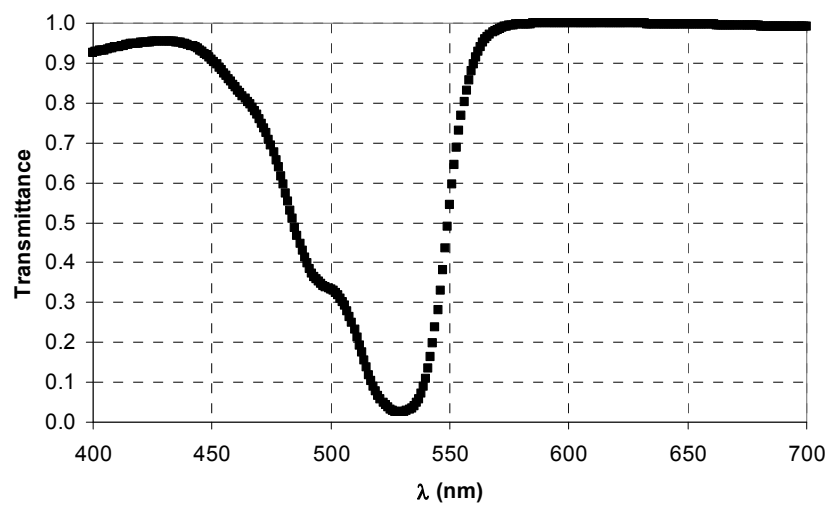

Figure 1. Transmittance of the unexposed photopolymer plate.

\subsection{Holographic set-up}

Reflection holographic gratings were stored using a Nd:YV04 laser (Coherent Verdi V2) with a wavelength of $532 \mathrm{~nm}$, to which the material was sensitive (see figure 1). A symmetric geometry was used in order to obtain gratings with interference fringes parallel to the recording material. The set-up is shown in figure 2 . The polarized beam emitted by the laser was split into two beams with a beam-splitter. Each beam was expanded and filtered using a microscope objective and a pinhole. Then the beams passed through a series of lenses and diaphragms in order to obtain collimated beams with the desired diameter. The total intensity of the recording beams was $30 \mathrm{~mW} / \mathrm{cm}^{2}$ with an intensity ratio of 1:1. The two laser beams were spatially overlapped at the recording medium intersecting at an angle of $63.3^{\circ}$ (measured in air).

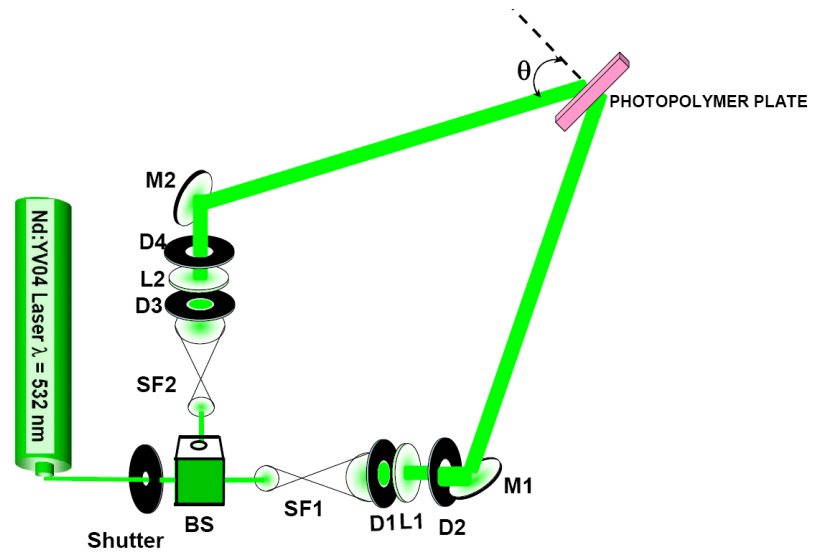

Figure 2. Experimental setup: BS beam splitter, Mi mirror, Li lens, Di, diaphragm, SFi, microscope objective lens and pinhole.

Using the value of the refractive index of the material obtained in the previous section, the intersection angle in air, $\theta$, and the recording wavelength, $\lambda=532 \mathrm{~nm}$, the theoretical spatial period of the grating, $\Lambda_{t h}$, was $0.2165 \mu \mathrm{m}$, applying equation (1) (therefore the spatial frequency of the recorded reflection gratings was 4618 lines $/ \mathrm{mm}$ ).

$$
\Lambda=\frac{\lambda}{2 \sqrt{n^{2}-\operatorname{Sin}^{2} \theta}}
$$




\section{RESULTS}

The main aim of this work is to study the stability of diffraction gratings stored holographically. To achieve this, holographic reflection gratings were stored according to the scheme of figure 2. Gratings with different exposures were recorded and diffraction efficiency was measured by a double-beam spectrophotometer.

The spectrometer measures the transmittance of the photopolymer versus the wavelength. When the grating is not stored in the photopolymer, the transmittance curve has a shape like that depicted in figure 1. However, an additional peak located at the Bragg wavelength appears after storing the grating, and its diffraction efficiency can be calculated from it. Fixing the spatial period of the grating, which as mentioned in the previous section is $\Lambda_{t h}=0.2165 \mu \mathrm{m}$, and knowing the refractive index of the material, according to equation 1, the Bragg wavelength will depend on the reconstruction angle, $\theta$. In figure 1, the absorption peak of the material appears around $530 \mathrm{~nm}$. However, from $590 \mathrm{~nm}$ the photopolymer has a high transmittance and it does not absorb anything. Therefore, if the grating was reconstructed with a suitable angle so that the diffraction peak had a Bragg wavelength of $590 \mathrm{~nm}$ or higher, it would not be overlapped with the absorption peak and it would facilitate the calculation of the diffraction efficiency of the grating. Therefore, the plate was placed perpendicular to the beam of the spectrophotometer $\left(\theta=0^{\circ}\right)$ with which the Bragg wavelength according to equation 1 , is $653 \mathrm{~nm}$. Once the grating has been stored in the material and it has been reconstructed with the spectrophotometer, at this wavelength is where the diffraction peak appears and we define the diffraction efficiency as the depth of this diffraction peak ${ }^{8,9}$ After storing the gratings in the material with different exposures, their transmittance was measured with the spectrophotometer following the described procedure.

It is important to record the gratings with the maximum diffraction efficiency as possible. However, when you are storing information, this information should remain over time. For this reason, in this work a curing process will be applied in order to fix the diffraction efficiency.

As mentioned in Section 2.1., one of the components of the photopolymer is a dye, the yellowish eosin, which absorbs photons, initiating the polymerization reaction. After recording the grating all the dye of the photopolymer has not been consumed yet, and the reaction continues but an uncontrolled manner, destroying the stored information and obtaining a diffraction efficiency equal to $0 \%$ after 24 hours. One way to avoid the uncontrolled reaction is remove the excess of dye after the recording. A curing process was used to eliminate the dye.

The instrument which was used for the curing process was an Aktiprint-M Compact UV Curing Bridge Unit. This device emits ultraviolet radiation with a power of $500 \mathrm{~W}$. It also has a conveyor belt where the recorded photopolymer is placed in order to receive the UV radiation during the time it takes to pass through the curing bridge. During that time period a certain amount of dye is removed. The more times the photopolymer goes through the curing bridge, more dye will be removed.

Figures 3 and 4 show the diffraction efficiency of the gratings recorded with different exposures before applying the curing process with black circles, just after applying the curing process with one pass through the curing bridge (figure 3 ) and 4 passes (figure 4 ) with red squares, and when 24 hours had passed after curing process with blue triangles.

Before applying the curing process, diffraction efficiency of the gratings have been represented in figures 3 and 4 with black circles versus the exposition with which have been stored. As it can be seen diffraction efficiency fluctuates from $4 \%$ for low exposures up to $6 \%$ for high exposures.

Just after applying the curing process, figure 3 shows the diffraction efficiency obtained with 1 pass in the curing bridge with red squares. The diffraction efficiency has been reduced approximately $50 \%$ compared with before of the applying curing process. However, 24 hours later the transmittance of the grating was re-measured with the spectrophotometer and the diffraction efficiency obtained is shown with blue triangles. As can be observed the diffraction efficiency has decreased slightly but it is not zero, as in the case without curing process. So the curing process has avoided the total destruction of the gratings. 
Cure: 1 Pass

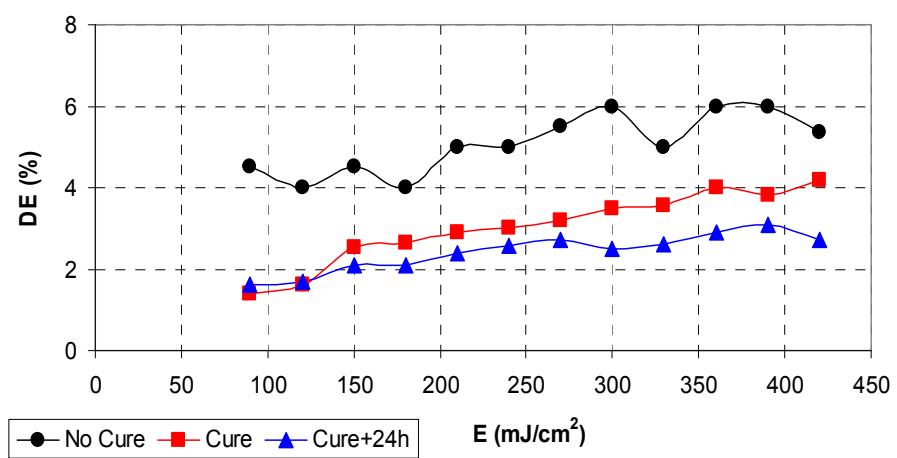

Figure 3. Diffraction efficiency versus exposure without cure process (black circles), after cure process (red squares) and 24 hours later of the cure process (blue triangles) for 1 pass in the curing bridge.

After that, gratings were recorded again in the photopolymer but now they were passed over the curing bridge 4 times to remove a greater amount of dye. Figure 4 shows, with red squares, the diffraction efficiency obtained versus the exposure. In this case the diffraction efficiency is slightly less than that obtained with a single pass through the curing. However 24 hours later, diffraction efficiency (blue triangles) has remained constant without any reduction.

Cure: 4 Pass

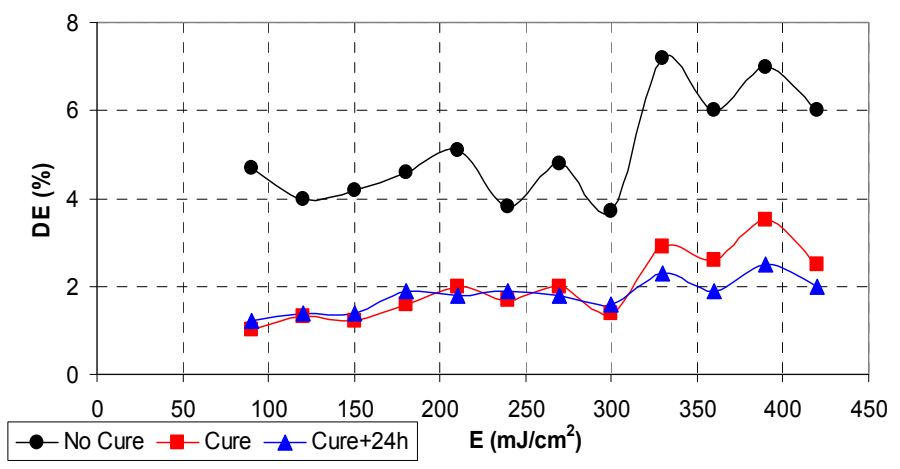

Figure 4. Diffraction efficiency versus exposure without cure process (black circles), after cure process (red squares) and 24 hours later of the cure process (blue triangles) for 4 passes in the curing bridge.

\section{CONCLUSIONS}

Holographic reflection gratings have been recorded with symmetric geometry in a PVA/AA based photopolymer. An element of the photopolymer is a dye that acts as an initiator in photopolymerization reactions. If the dye is not eliminated the grating will be degraded over time until reaching a diffraction efficiency equal to zero. To avoid this, a curing process is used in order to eliminate the excess of dye after the recording process. In this work it has been demonstrated how the total elimination of the dye through a curing process reduces the diffraction efficiency but it remains constant over time avoiding that information is completely eliminated. 


\section{ACKNOWLEDGMENTS}

This work was supported by "Ministerio de Ciencia e Innovación" (Spain) under projects FIS2011-29803-C02-01 and FIS2011-29803-C02-02, by the "Generalitat Valenciana" under project PROMETEO/2011/021 and by the University of Alicante under project GRE09-10.

\section{REFERENCES}

[1] Fernández, E., Ortuño, M., Gallego, S., García, C., Beléndez, A., Pascual, I., "Comparison of peristrophic multiplexing and a combination of angular and peristrophic holographic multiplexing in a thick PVA/acrylamide photopolymer for data storage," Appl. Opt. 46, 5368-5373, (2007).

[2] Fernández, E., Ortuño, M., Gallego, S., Márquez, A., García, C., Beléndez, A., Pascual, I., "Multiplexed holographic data page storage on a PVA/acrylamide photopolymer memory," Appl. Opt. 47, 4448-4456, (2008).

[3] Dhar, L., Curtis, K., Facke, T., "Holo graphic data storage: Coming of age," Nat. Photonics 2, 403-405, (2008).

[4] Graham-Rowe, D., "The drive for holography," Nat. Photonics 1, 197-200, (2007).

[5] Fernandez, E., Marquez, A., Gallego, S., Fuentes, R., García, C., Pascual, I., "Hybrid Ternary Modulation Applied to Multiplexing Holograms in Photopolymers for Data Page Storage," J. Lightwave Technol. 28, 776-783, (2010).

[6] Weiser, M., Bruder, F., Facke, T., Honel, D., Jurbergs, D., Rolle, T., "Self-Processing, Diffusion-Based Photopolymers for Holographic Apllications," Macromol. Symp. 296, 133-137, (2010).

[7] Ortuño, M., Gallego, S., García, C., Neipp, C., Beléndez, A., Pascual, I., "Optimization of a $1 \mathrm{~mm}$ thick PVA/acrylamide recording material to obtain holographic memories: method of preparation and holographic properties," Appl. Phys. B 76, 851-857, (2003).

[8] Fuentes, R., Fernandez, E., Garcia, C., Belendez, A., Pascual, I., "Study of reflection gratings recorded in polyvinyl alcohol/acrylamide-based photopolymer," Appl. Opt. 48, 6553-6557, (2009).

[9] Fuentes, R., Fernandez, E., Garcia, C., Belendez, A., Pascual, I., "Study of influence of ACPA in holographic reflection gratings recorded in PVA/AA based photopolymer," in Proc. of SPIE,7717, 77170Q-1-77170Q-11, (2010). 